\title{
INFECTION CONTROL AND HOSPITAL EPIDEMIOLOGY
}

\section{EDITORIAL}

Isolation Rooms for Tuberculosis Control

619

MARK NICAS, PHD, MPH, CIH; JOAN E. SPRINSON, MPH, CIH; SARAH E. ROYCE, MD, MPH;

ROBERT J. HARRISON, MD, MPH; J ANET M. MACHER, SCD, MPH

\section{ORIGINAL ARTICLES}

Evaluation of Rooms with N egative Pressure Ventilation Used for Respiratory Isolation in Seven M idwestern Hospitals

VICTORIA J. FRASER, MD; KRISTA JOHNSON; JONATHAN PRIMACK; MARILYN JONES, BSN; GERALD MEDOFF; W.C. DUNAGAN

A Case-Control Study of Nosocomial Ampicillin-Resistant Enterococcal Infection and Colonization at a University Hospital

DANIEL J. SEXTON, MD; LIZZIE J. HARRELL, PHD; JACKIE J. THORPE, MS, MT(ASCP);

DEBRA L. HUNT, DRPH; L. BARTH RELLER, MD

A N osocomial Pseudo-Outbreak of M ycobacterium xenopi Due to a

Contaminated Potable Water Supply: Lessons in Prevention

DAVID H. SNIADACK, MD; STEPHEN M. OSTROFF, MD; MICHAEL A. KARLIX, MS; RONALD W. SMITHWICK;

BENJ AMIN SCHWARTZ, MD; MARY ANN SPRAUER, MD, MPH; VELLA A. SILCOX; ROBERT C. GOOD, PHD

\section{BRIEF REPORT}

State Regulation of Hospital Water Temperature

ADAM S. MANDEL; MARY ANN SPRAUER, MD, MPH; DAVID H. SNIADACK, MD; STEPHEN M. OSTROFF, MD

\section{GLOBAL ASPECTS OF INFECTION CONTROL}

Global Aspects of Infection Control

MARY D. NETTLEMAN, MD, MS

\section{BEYOND INFECTION CONTROL:}

\section{THE NEW HOSPITAL EPIDEMIOLOGY}

The Use of Statistical Process Control Charts in Hospital Epidemiology

JOHN A. SELLICK, JR., DO

\section{PRODUCT COMMENTARY}

Needleless and N eedle Protection Devices: A Second Look at Efficacy

and Selection

ELIZABETH OWENS-SCHWAB, BSN; VICTORIA J. FRASER, MD

TOPICS IN CLINICAL MICROBIOLOGY

Hantavirus: An Old Bug Learns New Tricks

JOHN T. SINNOT IV, MD; JOHN N. GREENE, MD; EVELYN KIM, BA; SANDRA GOMPF, MD

\section{LETTERS TO THE EDITOR}

M olecular Epidemiology and Legionnaire's Disease

M. SABRIA-LEAL; M.L. PEDRO-BOTET; L. RUIZ; G. GIMENEZ

Nosocomial Infection Control in Latin America

MUSSARET ZAIDI, MD 


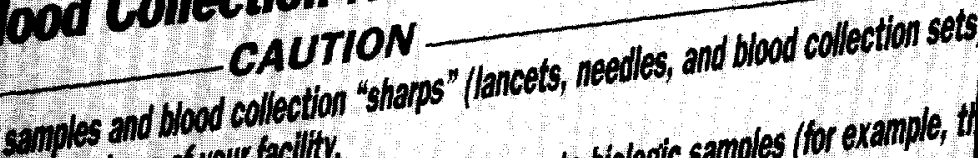
d procedures of your faclity.

wedical attention in the event of any exposure to biologic samples (for example, In. - in in protector, If the blood collection device provides one. Becton Dickinso 4. las, hut the poliches and procedures of your facility may difier andsal.

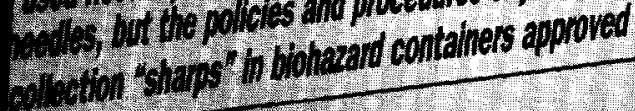

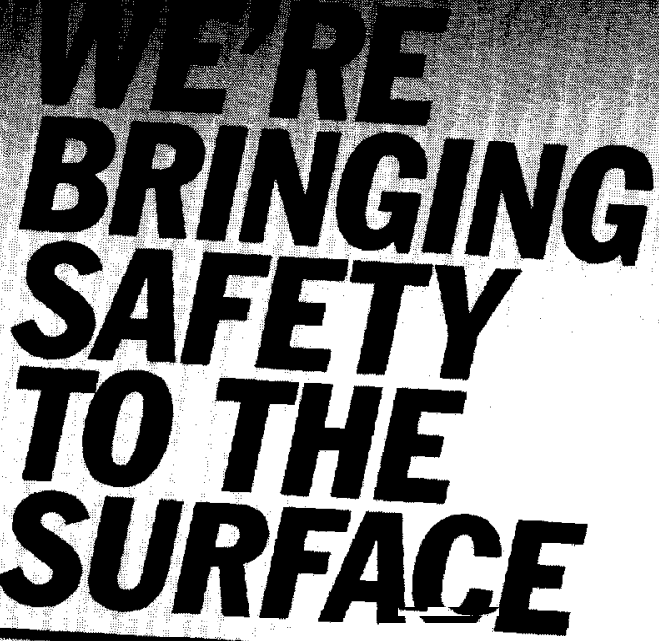

As the world's leading manufacturer of sharps products. workplace safer. Developing products that make today's healthcare regulations and setting new safety standards is with current commitment, but an equally important factor is a large part of that conscientious usage of all sharps products is promoting correct and accomplishing this goal, Becton products. As a step toward now including special safety messages on VACUTAINER Systems is packaging as an extra reminder to "handle with care."

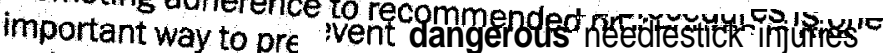
and exposure to haz ardous biologic specimens-and we at Becton Dickinson VACUTAINER Systems take this responsibility! seriously.

For detailed informat ion on all of our safety products contact your Becton I JickinsonVACUTAINERSystems ' Sales Representative.

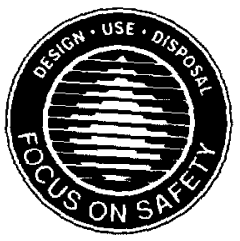

\section{BECTOM DICKISSON}




\section{INFECTION CONTROL AND Hospital EPIDEMIOLOGY}

\section{CONTENTS}

\section{EDITORIAL}

Isolation Rooms for Tuberculosis Control

MARK NICAS, PhD, MPH, CIH; JOAN E. SPRINSON, MPH, CIH; SARAH E. ROYCE, MD, MPH ROBERT J. HARRISON, MD, MPH; JANET M. MACHER, ScD, MPH

\section{ORIGINAL ARTICLES}

Evaluation of Rooms with Negative Pressure Ventilation Used for Respiratory Isolation in Seven Midwestern Hospitals

VICTORIA J. FRASER, MD; KRISTA JOHNSON; JONATHAN PRIMACK, MARILYN JONES, BSN; GERALD MEDOFF; W.C. DUNAGAN

A Case-Control Study of Nosocomial Ampicillin-Resistant Enterococcal Infection and Colonization at a University Hospital

DANIEL J. SEXTON, MD; STEPHEN M. OSTROFF, MD; MICHAEL A. KARLIX, MS; DEBRA L. HUNT, DrPH; L. BARTH RELLER, MD

A Nosocomial Pseudo-Outbreak of Mycobacterium xenopi Due to a Contaminated Potable Water Supply: Lessons in Prevention

DAVID H. SNIADACK, MD; STEPHEN M. OSTROFF, MD; MICHAEL A. KARLIX, MS; RONALD W. SMITHWICK; BENJAMIN SCHWARTZ, MD; MARY ANN SPRAUER, MD, MPH; VELLA A. SILCOX; ROBERT C. GOOD, PhD

BRIEF REPORT

State Regulation of Hospital Water Temperature

ADAM S, MANDEL; MARY ANN SPRAUER, MD, MPH; DAVID H. SNIADACK, MD;

STEPHEN M. OSTROFF, MD

GLOBAL ASPECTS OF INFECTION CONTROL

Global Aspects of Infection Control

MARY D. NETTLEMAN, MD, MS

BEYOND INFECTION CONTROL:

THE NEW HOSPITAL EPIDEMIOLOGY

The Use of Statistical Process Control Charts in Hospital Epidemiology

JOHN A. SELLICK, JR., DO

TOPICS IN CLINICAL MICROBIOLOGY

Hantavirus: An Old Bug Learns New Tricks

JOHN T. SINNOTT IV, MD; JOHN N. GREENE, MD; EVELYN KIM, BA; SANDRA GOMPF, MD

DE PAR T M E N T S

Letters to the Editor

Product Commentar

Calendar

666

Book Review 668

Medical News 670

SHEA News

675

The publication of advertising in the Journal does not constitute any guarantee or endorsement By The Society for Hospital EPidemiology

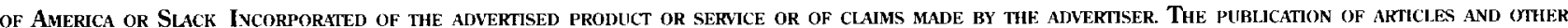
EDITORIAL MATERIAL IN THE JOURNAL DOES NOT NECESSARILY REPRESENT THE POLICY RECOMMENDATIONS OR ENDORSEMENT BY THE SOCIETY.

PuBuSHER: Infection Control and Hospital Epidemiology (ISSN-0899823X. Canadian GST\#129780466) is published exclusively by SLACK Incorporated 12 times a year. Address: 6900 Grove Rd., Thorofare. NJ 08086. Telephone:(609) 848-1000.

COPYRIGHT 1993 The Society for Hospital Epidemiology of America. Inc., and SIACK Incorporated. All rights reserved. No part of this publication may be reproduced without written permission from the publisher. Printed in the USA

SuBscriPTIONS: Requests should be addressed to the publisher (except Japan). In Japan. contact Woodbell Incorporated, 4-22-11, Kitakasai, Edogawaku, Tokyo 134. Japan. Subscription rates in the United States and possessions: Individual: One year- $\$ 80.00$; Two years- $\$ 115.00$; Three years-\$145.00; Institutional: One year- $\$ 90.00$; Two years-\$130.00; Three years-S170.00. Fellows: \$20 per year with proof of training status. Canada: \$18.00 additional each year plus $7 \%$ for Canadian Goods \& Services tax: all other countries: $\$ 36.00$ additional each year Single copies of current issues may be obtained for $\$ 15.00$, United States and possessions: $\$ 20.00$ all other countries.

REPRINTS: All requests to reprint or use material published herein should be addressed to Lester Robeson. SLACK Incorporated. 6900 Grove Rd., Thorofare NJ 08086. For reprint orders and prices. contact lester Robeson at (609) 84X-1000. Authorization to photocopy items for internal or personal use. or thr internal or personal use of specific clients. is granted by SLACK Incorporated, provided that the base fee of $\$ 1.00$ per copy, plus \$15 per page is paid directly to Copyright Clearance Center, 27 Congress St. Salem, MA01970. This consent does not extend to other kinds of copying. such as for general distribution, resale, advertising and promotional purposes, or for crating new collective works.

CHANGE OF ADDRESS: Notice should be sent to the publisher six weeks in advance of effective date. Include old and new addresses with zip codes. The publisher cannot accept responsibility for undelivered copies. Second-class postage is paid at Thorofare, Vew Jersey 08086, and additional entry points PostMaster: Send address changes to SL.ACK Incorporated, 6900 Grove Rd., Thorofare. NJ 08086.

INFECTION CONTROL AND HOSPITAL EPIDEMIOLOGY is listed in Index Medicos, Current Contents-Clinical Practice. Hospital Ijtorature Index, Cumulative Index to Nursing \&. Allied Health Literature. Nursing Abstracts, and Laboratory Performance Information Exchange System. 
EDITORIAL OFFICES

Vanderbilt University School of M edicine A-1131 M edical Center N orth

Nashville, TN 37232-2637

(615) 343-1095; (615) 343-1882 (FAX)

Email: iche@mcmail.vanderbilt.edu

EDITOR

M ichael D. Decker, MD, MPH

MANAGING EDITOR

Susan Cantrell

STATISTICAL EDITOR

Beverly G. M ellen, PhD

SENIOR ASSOCIATE EDITORS

C. Glen M ayhall, M D

Gina Pugliese, RN, MS

William Schaffner, M D

ASSOCIATE EDITORS

Donald A. Goldmann, M D

Didier Pittet, MD, MS

Andreas Widmer, MD, MS

SECTION EDITORS

Beyond Infection Control:

The New Hospital Epidemiology

Bryan P. Simmons, M D

Stephen B. Kritchevsky, PhD

M emphis, Tennessee

Wing Hong Seto, M D

Hong Kong

Disinfection and Sterilization

William A. Rutala, PhD, M PH

Chapel Hill, North Carolina

Emerging Infectious Diseases

Larry J. Strausbaugh, M D

Portland, Oregon

Robert W. Pinner, M D

Atlanta, Georgia

From the Laboratory

M arcus Zer vos, M D

Royal Oak, M ichigan

Fred C. Tenover, PhD

Atlanta, Georgia

Information Management

John A. Sellick, DO

Buffalo, N ew York

The International Perspective

M ary D. Nettleman, M D, MS

Richmond, Virginia

Issues in Surgery

James T. Lee, MD, PhD

St. Paul, M innesota

Medical News

Gina Pugliese, RN, M S

Chicago, Illinois

M artin S. Favero, PhD

Irvine, California

Practical Healthcare Epidemiology

Loreen A. Herwaldt, M D

lowa City, Iowa

SHEA News

M urray D. Batt, M D

Clarksburg, West Virginia

Statistics for Hospital Epidemiology

David Birnbaum, PhD, M PH

Sidney, British Columbia, Canada

Topics in Long-Term Care

Philip W. Smith, M D

Omaha, Nebraska

Topics in Occupational Medicine

David Weber, M D, M PH

Chapel Hill, North Carolina

\section{INFECTION CONTROL AND HOSPITAL EPIDEMIOLOGY \\ EDITORIAL ADVISORY BOARD}

Jacques F. Acar, MD Paris, France

J. Wesley Alexander, MD Cincinnati, Ohio Paul Arnow, M D Chicago, Illinois

Graham A.J. Ayliffe, MD Birmingham, United Kingdom Neil L. Barg, MD Yakima, Washington

Elizabeth Ann Bolyard, RN, M PH, CIC John M. Boyce, M D

Professor Dr. Ilja Braveny Charles Bryan, MD

Christian Brun-Buisson, M D

Donald $\mathrm{E}$. Craven, $\mathrm{MD}$

Sue Crow, M SN, RN, CIC

Franz Daschner, M D

Leigh G. Donowitz, MD

Charles E. Edmiston, Jr., PhD

Theodore C. Eickhoff, MD Bruce Farber, MD

Victoria J. Fraser, M D

Peter C. Fuchs, MD, PhD

Richard A. Garibaldi, MD

Velvl Greene, PhD, M PH Robert Gaynes, M D

David W. Gregory, M D

David K. Henderson, MD

Peter N.R. Heseltine, MD

Karen Hoffmann, RN, CIC, MS

$M$ arguerite $M$ CM illan Jackson, RN, PhD

Janine Jagger, M PH, PhD William R. Jar vis, M D

Douglas S. Kernodle, M D

Robert H. Latham, MD

Lewis B. Lefkowitz, MD

Hsieh-Shong Leu, M D, M Sc Jack Levy, MD

Victor Lorian, M D

Dennis G. M aki, M D

Professor Dr. Walter M arget

William J. M artone, M D

Allison M cGeer, MD

John E. M CGowan, Jr., M D

Jonathan L. M eakins, MD, DSC Raf M ertens, M D

Robert R. M uder, MD

Joseph M. M ylotte, M D, CIC Lindsay Nicolle, M D

J uhani Ojajär vi, M D

M ichael T. Osterholm, PhD, M PH

Jan Evans Patterson, M D Sindy M Paul, MD

M ichael A. Pfaller, MD

Samuel Ponce de Leon, M D, M Sc Isaam Raad, M D

M anfred L. Rotter, M D, DipBact Theodore Sacks, M D

William E. Scheckler, M D Kent Sepkowitz, M D Denis Spelman, M D

M ichael L. Tapper, M D

Clyde Thornsberry, PhD

Professor Leonid P. Titov

Timothy R. Townsend, M D

Antoni Trilla, MD, PhD

Professor Wang Shu-Qun

J. J ohn Weems, J r., M D

Robert A. Weinstein, M D

Professor Dr. W. Weuffen

Sergio B. Wey, MD Rebecca Wurtz, M D
Atlanta, Georgia

Providence, Rhode Island

M unich, Federal Republic of Germany

Columbia, South Carolina

Creteil, France

Boston, M assachusetts

Shreveport, Louisiana

Freiburg, Federal Republic of Germany

Charlottesville, Virginia

Milwaukee, Wisconsin

Denver, Colorado

$M$ anhasset, New York

St. Louis, M issouri

Black Butte, Oregon

Farmington, Connecticut

Beer Sheva, Israel

Atlanta, Georgia

Nashville, Tennessee

Bethesda, $\mathrm{M}$ aryland

Los Angeles, California

Chapel Hill, North Carolina

San Diego, California

Charlottesville, Virginia

Atlanta, Georgia

Nashville, Tennessee

Taipei, Taiwan

Brussels, Belgium

Bronx, New York

Madison, Wisconsin

M unich, Federal Republic of Germany

Bethesda, $\mathrm{M}$ aryland

Toronto, Ontario, Canada

Atlanta, Georgia

M ontreal, Quebec, Canada

Brussels, Belgium

Pittsburgh, Pennsylvania

Buffalo, N ew York

Winnepeg, $\mathrm{M}$ anitoba, Canada

Helsinki, Finland

M inneapolis, M innesota

San Antonio, Texas

Trenton, N ew Jersey

lowa City, Iowa

M exico City, M exico

Houston, Texas

Vienna, Austria

Jerusalem, Israel

$M$ adison, $W$ isconsin

New York City, New York

Prahran Victoria, Australia

New York, New York

Brentwood, Tennessee

M insk, Republic of Belarus

Millwood, Virginia

Barcelona, Spain

B eijing, People's Republic of China

Greenville, South Carolina

Chicago, Illinois

Greifswald, Federal Republic of Germany

São Paulo, Brazil

Evanston, Illinois
Nashville, Tennessee

Nashville, Tennessee

\begin{tabular}{|c|c|c|}
\hline \multicolumn{3}{|c|}{$\begin{array}{c}\text { SLACK Incorporated } \\
6900 \text { Grove Road } \\
\text { Thorofare, N ew Jersey } 08086 \\
\text { (609) } 848-1000\end{array}$} \\
\hline $\begin{array}{l}\text { Vice President/ Group Publisher } \\
\text { Richard N. Roash }\end{array}$ & $\begin{array}{l}\text { Assistant E ditor } \\
\text { Eileen C. Anderer }\end{array}$ & $\begin{array}{l}\text { Publishing Director/ Advertising } \\
\text { Wayne M CC ourt }\end{array}$ \\
\hline $\begin{array}{l}\text { Publisher } \\
\text { John C. Carter }\end{array}$ & $\begin{array}{l}\text { Circulation Manager } \\
\text { Lester J. Robeson, CССР }\end{array}$ & $\begin{array}{l}\text { Pharmaceutical Group Sales Director } \\
\text { M ichael LoPresti }\end{array}$ \\
\hline $\begin{array}{l}\text { E ditorial Director } \\
\text { Jennifer Kilpatrick }\end{array}$ & $\begin{array}{l}\text { Production Director } \\
\text { Christine M alin }\end{array}$ & $\begin{array}{l}\text { Advertising Sales Representative } \\
\text { Jennine Kane }\end{array}$ \\
\hline $\begin{array}{l}\text { Production E ditor } \\
\text { Shirley P. Strunk, ELS }\end{array}$ & $\begin{array}{l}\text { Production Coordinator } \\
\text { J oanne Patterson }\end{array}$ & $\begin{array}{l}\text { Classified/ Recruitment Sales Manager } \\
\text { M ichele Burch }\end{array}$ \\
\hline
\end{tabular}




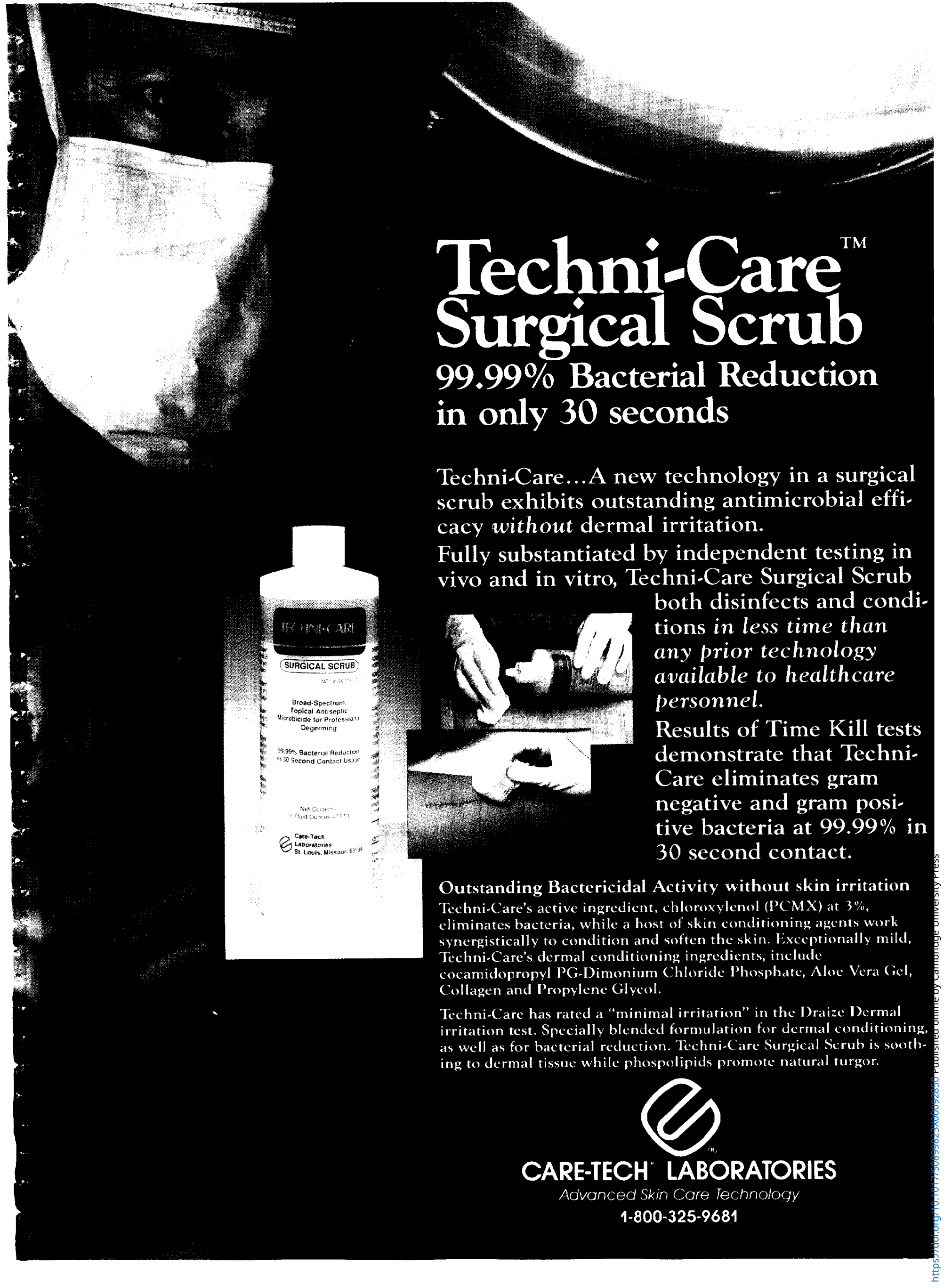

